\title{
Bilevel optimization techniques in computational strain design
}

\author{
Anupam Chowdhury ${ }^{1}$, Ali R. Zomorrodi ${ }^{2}$ and Costas D. Maranas ${ }^{1 *}$ \\ ${ }^{1}$ Department of Chemical Engineering, The Pennsylvania State University, University Park, \\ University Park, PA 16802 \\ ${ }^{2}$ Program in Bioinformatics, Boston University, Boston MA 02215 \\ *Corresponding author \\ E-mail: costas@psu.edu \\ Phone: 814-863-9958
}




\begin{abstract}
Over the past decade a number of bilevel optimization techniques were introduced for computational strain design leading to the overproduction of biochemicals. In this paper, we provide an algorithm-centric description of the OptKnock and OptForce strain-design protocols, highlight their differences and demonstrate their application for a prototypical overproduction problem. The derivation of the equivalent MILP representation in both cases is described in detail along with provisions that lead to significantly improved performance. Comparison between the intervention strategies of OptKnock and OptForce for the overproduction of succinate in $E$. coli reveal that while OptKnock couples succinate with biomass production, OptForce suggests interventions that improves the minimum production of succinate. Further, OptForce is more tractable as it identifies interventions from only the subset of reactions that must change in the overproducing strain. Overall, this paper highlights the computational challenges faced in strain design and the methodological choices explored by OptKnock and OptForce.
\end{abstract}

Keywords: computational strain-design, bilevel problem, MILP formulation 


\section{Introduction}

Over the past decade, there has been rapid progress in the development of MILP and MINLP algorithmic techniques for the analysis and redesign of metabolic networks (Burgard et al., 2003; Cotten \& Reed, 2013; J. Kim \& Reed, 2010; J. Kim et al., 2011; Patil et al., 2005; Pharkya et al., 2004; Pharkya \& Maranas, 2006; Yang et al., 2011). While tasks in metabolic networks differ from process systems engineering problems, the same underlying algorithmic challenges are encountered ranging from nonlinearities in description, parametric uncertainty and combinatorial explosion. In metabolism, genome-scale models of metabolism describe the complex network of enzyme-catalyzed reactions encoded by the genome of an organism. These reactions are responsible for a wide variety of functions in a given microorganism such as uptake/export of metabolites into and out of the cell, synthesis and degradation of cellular growth precursors (e.g., amino acids, cofactors), as well as maintaining the energy requirements and redox balance required to sustain cell viability. With recent advances in genome-sequencing and annotation techniques (Macaulay \& Voet, 2014; Yu et al., 2013), there has been a steady increase in the number and diversity of genome-scale model reconstructions (T. Y. Kim et al., 2012) ranging from unicellular to multi-cellular to multi-tissue organisms. These models have been extensively used not only for analyzing the metabolic capability of a given microorganism but also for modeldriven discovery of missing functions, hypothesis generation/validation (McCloskey et al., 2013) and for a wide array of biomedical and biotechnological applications.

Genome-scale metabolic models coupled with conservation of mass equations for each metabolite in the network provide the quantitative means to infer the metabolic capabilities of a given organism:

$$
\frac{d c_{i}}{d t}=\sum_{j \in J} S_{i j} v_{j}, \quad \forall i \in I
$$

Here, $\boldsymbol{I}$ and $\boldsymbol{J}$ are the sets of metabolites and reactions, respectively, describing the metabolite concentrations $C_{i}$ and reaction fluxes $v_{j}$. $S_{i j}$ describe the stoichiometric coefficient of metabolite $i$ in reaction $j$. Considering the widely different time-scale of different cellular processes it is a common practice to assume a "pseudo steady-state" inside the cell (Orth et al., 2010) reducing equation above to:

$$
\sum_{j \in J} S_{i j} v_{j}=0, \quad \forall i \in I
$$


Given that the number of reactions is higher than the number of metabolites in a metabolic network the above system of equations is under-determined implying that there can be infinite number of reaction fluxes that satisfy the steady-state mass balance equation. Therefore, one needs to select from this infinite number of solutions only those that closely represent the physiological conditions inside the cell. This is typically achieved by solving a Linear Programming (LP) problem that maximizes the flux of the biomass reaction (a reaction whose flux approximates all cellular components required for growth in their appropriate biological ratios) subject to steady-state mass balance equation and specified lower $\left(L B_{j}\right)$ and upper bounds $\left(U B_{j}\right)$ on reaction fluxes:

$$
\begin{array}{ll}
\underset{v_{j}}{\operatorname{maximize}} \quad v_{\text {biomass }} & \\
\text { subject to } & \\
& \sum_{j \in J} S_{i j} v_{j}=0, \quad \forall i \in I \\
& L B_{j} \leq v_{j} \leq U B_{j}, \quad \forall j \in J \\
& v_{j} \in \mathfrak{R}
\end{array}
$$

This LP is the basis of Flux Balance Analysis (FBA) (Varma \& Palsson, 1994) and has been used extensively for analyzing the metabolic potential of genome-scale models (Orth et al., 2010) only using stoichiometric information. Recently, additional constraints of fluxomic (Crown \& Antoniewicz, 2013; Zomorrodi et al., 2012) and/or transcriptomic (Schmidt et al., 2013) information have been incorporated in FBA to further improve the characterization of metabolic phenotype (Saha et al., 2014). In addition, the incorporation of kinetic information onto genomescale models is actively explored by many groups (Chowdhury et al., 2014; Cotten \& Reed, 2013; Famili et al., 2005; Feng et al., 2012; Fleming et al., 2010; Zomorrodi et al., 2014).

FBA has found extensive use in the metabolic engineering of microorganisms where the aim is to rewire the metabolic network in order to direct more metabolic flux towards a biochemical of interest (McCloskey et al., 2013). A number of optimization-based frameworks using FBA as the basis have been developed in the past decade to identify the trade-off between the yield of a desired chemical and biomass production (Cintolesi et al., 2014), genetic knock-ins for heterologous expression of a metabolic function (J. Kim et al., 2011; Pharkya et al., 2004) and to predict the new phenotype in the event of a genetic deletion (Segre et al., 2002). The use of bilevel optimization was ushered by the strain design algorithm called OptKnock (Burgard et al., 2003). The key concept here is to use an inner problem that re-apportions fluxes in the network in 
response to gene knock-outs so as to maximize biomass formation and an outer level that optimizes the production of the biochemical of interest by identifying appropriate gene knock-out (Ibarra et al., 2002; Segre et al., 2002). Ultimately, this establishes a way of computationally enforcing the requirement for a targeted overproduction as a requirement for growth. This has had far reaching implications in strain selection and stability. Binary variables are used to capture the removal of a reaction (i.e., gene knock-out) by collapsing the lower and upper bounds of the corresponding flux in the inner problem to zero. Alternatively, approaches such as BiMOMA (J. Kim et al., 2011) have been developed that use the minimization of the metabolic adjustment (MOMA) (Segre et al., 2002) as the cellular objective instead of maximization of the biomass. The OptForce algorithm (Ranganathan et al., 2010), on the other hand, assumes a conservative "worst-case" scenario by minimizing the flux of the desired chemical as the cellular response. Most other constraint-based protocols (J. Kim \& Reed, 2010; Patil et al., 2005; Rocha et al., 2010) predict the flux distribution of the perturbed network using one or a combination of these assumptions. These bilevel optimization based procedures have been successfully employed for the overproduction of several important chemicals and biofuel precursors (Alper et al., 2005; Asadollahi et al., 2009; Bro et al., 2006; Choi et al., 2010; Lee et al., 2005; Park et al., 2007), the analysis and curation of metabolic networks (Kumar \& Maranas, 2009; Suthers et al., 2009) and microbial communities (Zomorrodi et al., 2014; Zomorrodi \& Maranas, 2012).

Despite the availability of a long list of strain design procedures in literature, a comparative study of the merits and demerits of these approaches is still lacking. In this study, we aim to address this gap by using OptKnock (Burgard et al., 2003) and OptForce (Ranganathan et al., 2010) as two prototype strain design procedures. We start by presenting the stepwise formulations of these two procedures along with a systematic solution procedure of the mixed-integer bilevel optimization structures exploiting the primal and dual structures. This is followed by a comparative study of the computational performance of these two procedures along with suggested ways of improving their computational efficiency with succinate overproduction in E. coli as a case study. In addition, differences in the engineering strategies identified by each framework, which is due to the differing nature of allowed interventions and optimality posture for the inner problem, are discussed in the context of succinate overproduction. These analyses revealed that as the number of interventions increased OptForce requires significantly less computational time compared to OptKnock as it searches within a small subset of reactions that are most relevant to the desired overproduction. Furthermore, while interventions suggested by OptKnock couple the succinate production with biomass, those identified by OptForce force flux toward succinate while keeping 
biomass

formation

level. 


\section{Methods}

Here, we describe the detailed mathematical formulations of the OptKnock and OptForce procedures as refined in practice over the past few years. This treatment builds upon the original introduction of the methods by carefully delineating all computational steps, problem reformulation and solution strategies found to be the most efficient in practice. It provides a number of unconventional applications of bilevel and MILP optimization in an area far removed from process systems engineering where these mathematical techniques were refined.

\subsection{OptKnock}

OptKnock is a computational strain design procedure that aims to identify minimum sets of reaction removals in a metabolic network coupling the production of a target chemical with cellular biomass formation. Reaction removals are enforced by defining a binary variable as follows:

$\mathrm{y}_{j}=\left\{\begin{array}{l}1, \text { if reaction is removed } \\ 0, \text { otherwise }\end{array}\right.$

The OptKnock procedure is then mathematically described by the following bilevel optimization problem:

$\underset{y_{j}}{\operatorname{maximize}} \quad v_{\text {prod }}$
subject to

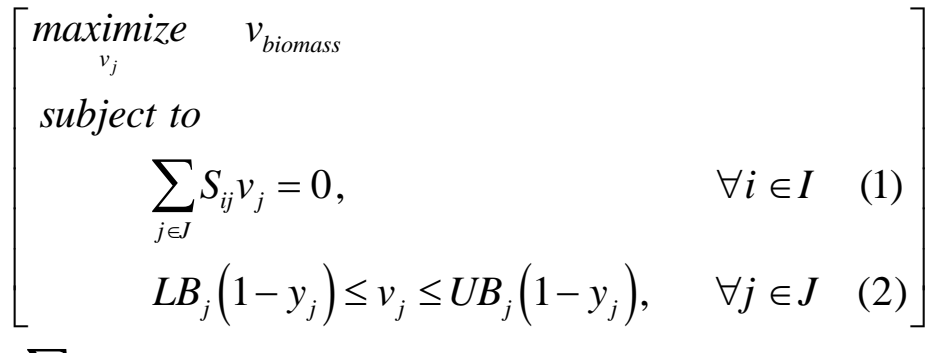

$$
\begin{aligned}
& \sum_{j \in J} y_{j}=\kappa, \\
& y_{j} \in\{0,1\}, v_{j} \in \mathfrak{R}, \quad \forall j \in J
\end{aligned}
$$


The outer problem identifies optimal reaction removals (imposed by using the binary variables $\left.y_{j}\right)$ that maximize the flux through a chemical of interest $\left(v_{\text {prod }}\right)$. The inner problem identifies the flux distribution maximizing the cellular objective $\left(v_{\text {biomass }}\right)$ subject to the reaction removals imposed by the outer problem. Constraint (1) describes the steady-state conservation of mass for each metabolite in the network and Constraint (2) forces the flux of a reaction to zero if its corresponding binary variable is one. The total number of reaction removals is also fixed at a prespecified value $\kappa$ by using Constraint (3).

The lower- and upper-bound on reaction fluxes $\left(L B_{j}\right.$ and $\left.U B_{j}\right)$ are defined as follows for each type of reaction:

$$
\begin{aligned}
& L B_{j}= \begin{cases}v_{\text {biomass }}^{\text {min }}, & \text { for } j \in\{\text { biomass }\} \\
v_{\text {atpm }}^{\text {min }}, & \text { for } j \in\{\text { ATPM }\} \\
0, & \text { for } j \in J^{\text {irrev }} \backslash\{\text { biomass, ATPM }\} \\
-M, & \text { for } j \in J^{\text {rev }} \\
-v_{j}^{\text {uptake }}, & \text { for } j \in J^{\text {uptake }} \\
0, & \text { for } j \in J^{\text {exch }} \backslash J^{\text {uptake }}\end{cases} \\
& U B_{j}= \begin{cases}v_{a t p m}^{\mathrm{min}}, & \text { for } j \in\{\mathrm{ATPM}\} \\
M, & \text { for } j \in J \backslash J^{\text {uptake }} \cup\{\mathrm{ATPM}\}\end{cases}
\end{aligned}
$$

Reversible reactions $J^{\text {rev }}$ are not restricted in sign ( $M$ is a big number), while irreversible reactions can only take a non-negative flux value. It is common practice to add a class of ad-hoc reversible reactions that model the transport of metabolites into and out of the cell. These reactions are called "exchange” reactions $J^{\text {exch }}$ where the forward direction represents export and the reverse direction uptake of metabolites. It is typically assumed that all metabolites for which an exchange reaction exists in the model can be freely exported out of the cell and thus the upper bounds for all exchange reactions is set to a large positive value (e.g., $M$ ). In contrast, only the nutrients that are available in the growth medium ( $J^{\text {uptake }}$ ) are allowed to be taken up by the cell and thus the lower bound for these compounds are set to a negative value. For nutrients available in excess in the medium, this lower bound is set to $-M$, while for limiting resources (such as carbon source and oxygen) it is set to a pre-defined value. The flux of non-growth associated maintenance (ATPM) is also set to its experimentally measured value $v_{a t p m}^{\min }$. In addition, the 
lower bound on the flux of biomass reaction (representing the cell's growth) is set to a nonnegative value (e.g., $10 \%$ of theoretical maximum) to ensure viability.

Starting from a relatively low number of interventions, OptKnock is rerun iteratively by successively increasing $\kappa$ to explore the impact of additional interventions on improving product yield. This allows a natural prioritization of the interventions, where the interventions having the largest impact on improving the product yield are identified first. Integer cuts are used to identify alternate interventions as well as to explore the sub-optimal space of knock-outs for a given value of $\kappa$. They are imposed by addition of the following constraint to the outer problem of OptKnock:

$$
\sum_{j \mid y_{j}^{k}=0} y_{j}+\sum_{j \mid y_{j}^{k}=1}\left(1-y_{j}\right) \geq 1 \quad \forall k=1,2, \ldots, K
$$

where $K$ is the total number of explored solutions so far and $y_{j}^{k}$ stores the selected interventions of the $k^{\text {th }}$ solution identified previously.

The bilevel problem of OptKnock is solved by converting it into a single-level MILP by augmenting the constraints of the outer and inner problem with the dual constraints of the inner problem and imposing the strong duality condition. The dual of the inner problem can be written as follows:

$$
\begin{array}{cl}
\operatorname{minimize}_{\lambda_{i}, \mu_{j}^{L B}, \mu_{j}^{U B}} & \sum_{j \in J} \mu_{j}^{U B} U B_{j}\left(1-y_{j}\right)-\sum_{j \in J} \mu_{j}^{L B} L B_{j}\left(1-y_{j}\right) \\
\text { subject to } & \\
\sum_{i \in I} \lambda_{i} S_{i j}+\mu_{j}^{U B}-\mu_{j}^{L B}=1, & \forall j \in\{\text { biomass }\} \\
\sum_{i \in I} \lambda_{i} S_{i j}+\mu_{j}^{U B}-\mu_{j}^{L B}=0, & \forall j \in J \backslash\{\text { biomass }\} \\
\mu_{j}^{L B}, \mu_{j}^{U B} \geq 0, & \forall j \in J \\
\lambda_{i} \in \mathfrak{R}, & \forall i \in I
\end{array}
$$

where dual variables for the primal constraints are defined as follows: 


$$
\begin{array}{lll}
\sum_{j \in J} S_{i j} v_{j}=0 & \forall i \in I & \rightarrow \lambda_{i}, \text { where } \lambda_{i} \in \mathfrak{R} \\
-v_{j} \leq-L B_{j}\left(1-y_{j}\right) & \forall j \in J & \rightarrow \mu_{j}^{L B}, \text { where } \mu_{j}^{L B} \geq 0 \\
v_{j} \leq U B_{j}\left(1-y_{j}\right) & \forall j \in J & \rightarrow \mu_{j}^{U B}, \text { where } \mu_{j}^{U B} \geq 0
\end{array}
$$

Note that the binary variables $y_{j}$ are variables of the outer problem but are treated as parameters for the inner problem.

The bilinear terms in the objective function of the dual problem can be recast in the form of equivalent linear relations using the available standard techniques (Glover, 1975). An analysis of all the terms in the optimization formulation can further reduce the number of variables the problem. In particular, terms $L B_{j} \mu_{j}^{L B}\left(1-y_{j}\right)$ and $U B_{j} \mu_{j}^{U B}\left(1-y_{j}\right)$ can be removed if the binary variable $y_{j}$ assumes a value of one (i.e., knock-out).

$$
y_{j}=1 \rightarrow \begin{cases}L B_{j} \mu_{j}^{L B}\left(1-y_{j}\right)=0 & \forall j \in J \\ U B_{j} \mu_{j}^{U B}\left(1-y_{j}\right)=0 & \forall j \in J\end{cases}
$$

Likewise, the expressions $L B_{j} \mu_{j}^{L B}\left(1-y_{j}\right)$ and $U B_{j} \mu_{j}^{U B}\left(1-y_{j}\right)$ are reduced to $L B_{j} \mu_{j}^{L B}$ and $U B_{j} \mu_{j}^{U B}$, respectively, if the binary variable assumes a value of zero $\left(y_{j}=0\right)$. Furthermore, the complementary slackness conditions set $\mu_{j}^{L B}$ and/or $\mu_{j}^{U B}$ to zero for reactions whose corresponding primal problem constraints are inactive (i.e. reactions for which $L B_{j}=-M$ and $U B_{j}=M$ respectively, where, $M$ is a big number). This eliminates the term $L B_{j} \mu_{j}^{L B}\left(1-y_{j}\right)$ for reactions except for irreversible reactions ( $J^{\text {irrev }}$ ), biomass formation, ATPM and exchange reactions corresponding to limiting compounds in the growth medium (e.g., carbon source, oxygen, collectively contained in set $\left.J^{\text {exch,limiting }}\right)$. Correspondingly term $U B_{j} \mu_{j}^{U B}\left(1-y_{j}\right)$ is eliminated for all reactions except for ATPM.

$$
y_{j}=0 \rightarrow \begin{cases}L B_{j} \mu_{j}^{L B}\left(1-y_{j}\right)=0 & \forall j \in J \backslash J^{\text {irrev }} \cup J^{\text {exch,limiting }} \cup\{\text { biomass, ATPM }\} \\ L B_{j} \mu_{j}^{L B}\left(1-y_{j}\right)=L B_{j} \mu_{j}^{L B} & \forall j \in J^{\text {irrev }} \cup J^{\text {exch,limiting }} \cup\{\text { biomass, ATPM }\} \\ U B_{j} \mu_{j}^{U B}\left(1-y_{j}\right)=0 & \forall j \in J \backslash\{A T P M\} \\ U B_{j} \mu_{j}^{U B}\left(1-y_{j}\right)=U B_{j} \mu_{j}^{U B} & \forall j \in\{A T P M\}\end{cases}
$$


Therefore, the nonlinear objective function of the dual problem is reduced to the following linear form:

$$
Z=\mu_{\text {ATPM }}^{U B} U B_{\text {ATPM }}-\left(\mu_{\text {biomass }}^{L B} L B_{\text {biomass }}+\mu_{\text {ATPM }}^{L B} L B_{\text {ATPM }}+\sum_{j \in J^{\text {irrev }} \cup J^{\text {exch,limiting }}} \mu_{j}^{L B} L B_{j}\right)
$$

In addition, for reactions that are not selected for removal $\left(y_{j}=0\right)$, constraints must be added to ensure that the values of the dual variables $\mu_{j}^{L B}$ and $\mu_{j}^{U B}$ are equal to zero when the corresponding primal constraints for the bounds on fluxes are inactive.

$$
\begin{array}{ll}
0 \leq \mu_{j}^{L B} \leq \mu_{j}^{L B, \max } y_{j}, & \forall j \in\left\{j \mid L B_{j}=-M\right\} \\
0 \leq \mu_{j}^{U B} \leq \mu_{j}^{U B, \max } y_{j}, & \forall j \in\left\{j \mid U B_{j}=M\right\}
\end{array}
$$

Integrating the constraints of the primal and dual problem and imposing the strong duality condition, OptKnock can be recast as the following single-level MILP:

$$
\begin{aligned}
& \underset{y_{j}}{\operatorname{maximize}} \quad v_{\text {prod }} \\
& \text { subject to } \\
& v_{\text {biomass }}=\mu_{\text {ATPM }}^{U B} U B_{A T P M}-\left(\mu_{\text {biomass }}^{L B} L B_{\text {biomass }}+\mu_{\text {ATPM }}^{L B} L B_{\text {ATPM }}+\sum_{j \in J^{\text {irrev }} \cup J^{\text {exch limiting }}} \mu_{j}^{L B} L B_{j}\right) \\
& \sum_{j \in J} S_{i j} v_{j}=0, \quad \forall i \in I \\
& L B_{j}\left(1-y_{j}\right) \leq v_{j} \leq U B_{j}\left(1-y_{j}\right), \quad \forall j \in J \\
& \sum_{i \in I} \lambda_{i} S_{i j}+\mu_{j}^{U B}-\mu_{j}^{L B}=1, \quad \forall j \in\{\text { biomass }\} \\
& \sum_{i \in I} \lambda_{i} S_{i j}+\mu_{j}^{U B}-\mu_{j}^{L B}=0, \quad \forall j \in J \backslash\{\text { biomass }\} \\
& 0 \leq \mu_{j}^{L B} \leq \mu_{j}^{L B, \max } y_{j}, \quad \forall j \in\left\{j \mid L B_{j}=-M\right\} \\
& 0 \leq \mu_{j}^{U B} \leq \mu_{j}^{U B, \max } y_{j}, \quad \forall j \in\left\{j \mid U B_{j}=M\right\} \\
& \sum_{j \in J} y_{j}=\kappa, \\
& \lambda_{i} \in \mathfrak{R}, \quad \forall i \in I \\
& y_{j} \in\{0,1\}, \mu_{j}^{L B} \geq 0, \mu_{j}^{U B} \geq 0, v_{j} \in \mathfrak{R}, \quad \forall j \in J
\end{aligned}
$$

The number of binary variables can be reduced significantly by fixing those corresponding to reactions that are not allowed to be removed or their removal does not have any effect on the 
network. Examples of reactions in the former category are ATPM, biomass reaction, uptake reactions and essential reactions (i.e. reactions that must be active to ensure a non-zero biomass production). Blocked reactions (i.e., those that cannot carry any flux in a given growth medium) are also example of reactions whose removal does not affect the flux distribution in the network. Similarly to essential reactions, additional constraints can be added to the outer problem to avoid the simultaneous removal of synthetic lethal reaction pairs, triples etc. (Suthers et al., 2009)

\subsection{OptForce}

OptForce builds upon computational strain design procedures such as OptKnock and variants thereof (J. Kim \& Reed, 2010; Pharkya et al., 2004; Pharkya \& Maranas, 2006; Tepper \& Shlomi, 2010). In addition to reaction removals, OptForce identifies reaction up/down-regulations, while also taking into account the available metabolic flux data for the reference and/or mutant strains in its design framework (Ranganathan et al., 2010). The OptForce procedure is comprised of two main steps.

\subsubsection{Identification of the MUST sets}

Metabolic flux analysis (MFA) data are used first to characterize the phenotypic space of a "reference" strain by successively maximizing and minimizing the flux of each reaction in the network. These data are often available for only a small subset of reactions in the network (e.g., those in the central carbon metabolism) and are typically presented as a range, which are added as new constraints to the regular FBA problem:

$\left.\begin{array}{lll}\underset{v_{j}}{\operatorname{maximize} \text { minimize }} \quad v_{j} & \\ \text { subject to } & \forall i \in I & \text { (1) } \\ \sum_{j \in J} S_{i j} v_{j}=0, & \forall j \in J \backslash J^{M F A} & \text { (2) } \\ L B_{j} \leq v_{j} \leq U B_{j}, & \forall j \in J^{M F A} & \text { (3) } \\ v_{j}^{L B, M F A} \leq v_{j} \leq v_{j}^{L B, M F A}, & & \text { (4) } \\ v_{\text {biomass }} \geq v_{\text {biomass }}^{\max }, & \forall j \in J & \text { (5) } \\ v_{j} \in \mathfrak{R}, & & \end{array}\right\} \forall$

Here, the lower bound on the flux of biomass reaction is set to the maximum predicted flux $v_{\text {biomass }}^{\max }$ consistent with the MFA data (Constraint 3).

The next step in OptForce is to identify the flux ranges consistent with a desired overproduction target. This is done in a similar way by successively maximizing and minimizing the flux of 
network reactions subject to network stoichiometry, minimum biomass production $v_{\text {biomass }}^{\text {min }}$ (to ensure viability) and a desired flux of the target product:

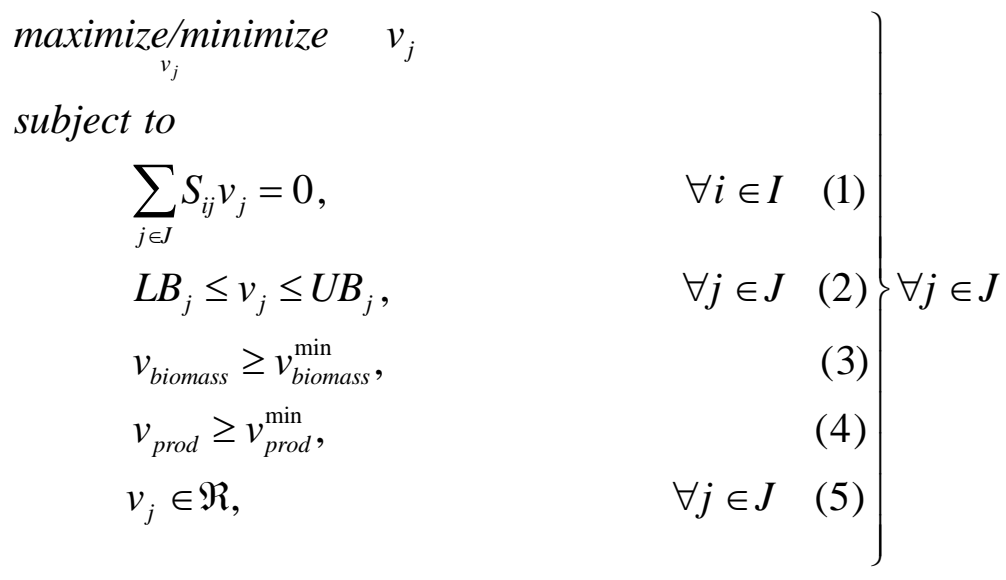

By superimposing the flux ranges for each reaction in the reference and overproducing strains the set of all reactions whose flux 'must' change to satisfy the overproduction target are identified. These reactions are referred to as MUST sets of reactions, which are categorized into MUST ${ }^{\mathrm{L}}$ and $\mathrm{MUST}^{\mathrm{U}}$ sets if the flux range in the reference strain lies totally to the left or to the right of the corresponding range in the overproducing strain, respectively. Reactions whose flux range collapses to zero in the overproducing strain are also categorized as MUST ${ }^{\mathrm{X}}$, implying that these reactions need to be shut off to achieve the overproduction target. The cases for which there is an overlap between the reference and overproducing flux ranges do not provide any information on the flux changes, however, they can be further scrutinized by iteratively maximizing the sum and/or difference of the fluxes of each pair of such reactions. By contrasting the identified ranges new MUST sets of reactions including MUST ${ }^{\mathrm{LU}}$, MUST ${ }^{\mathrm{UU}}$ and MUST ${ }^{\mathrm{LL}}$ (i.e., MUST pairs) can be identified. For example, if a reaction pair belongs to set $\mathrm{MUST}^{\mathrm{UU}}$, it implies that flux of either one of these reactions (but not necessarily both) has to be increased to achieve the desired overproduction target. The exhaustive identification of MUST pairs and higher order sets (e.g., triples, quadruples, etc.) rapidly becomes computationally intractable, and thus a bilevel optimization structure was proposed for the targeted identification of MUST sets (Ranganathan et al., 2010). For example, for a reaction pair $\left(j_{1}, j_{2}\right)$ to belong to $\mathrm{MUST}^{\mathrm{LU}}$ set it is needed to have $\max \left(v_{j_{1}}^{o s}-v_{j_{2}}^{o s}\right)<\min \left(v_{j_{1}}^{r e f}-v_{j_{2}}^{r e f}\right)$ as the flux ranges of $v_{j_{1}}-v_{j_{2}}$ in the reference and overproducing strains should not overlap (superscripts 'os' and 'ref' indicate overproducing and 
reference strains, respectively). Two binary variables are defined as follows to encode whether a reaction $\left(j_{1}, j_{2}\right)$ belong to MUST ${ }^{\mathrm{LU}}$ sets:

$\mathrm{y}_{j 1}^{L}=\left\{\begin{array}{l}1, \text { if reaction } \mathrm{j}_{1} \text { isidentified for down-regulation in a } \mathrm{MUST}^{\mathrm{LU}} \text { pair } \\ 0 \text {, otherwise }\end{array}\right.$

$\mathrm{y}_{j 2}^{U}=\left\{\begin{array}{l}1, \text { if reaction } \mathrm{j}_{2} \text { is identified for up-regulation in a MUST }{ }^{\mathrm{LU}} \text { pair } \\ 0, \text { otherwise }\end{array}\right.$

The bilevel optimization problem for the identification of MUST ${ }^{\mathrm{LU}}$ set of reactions can then be formulated as follows:

$$
\begin{aligned}
& \underset{y_{j 1}^{L}, y_{j 2}^{U}}{\operatorname{maximize}} \quad Z=\sum_{j \in J}\left(v_{j 1}^{r e f, L} y_{j 1}^{L}-v_{j 2}^{r e f, U} y_{j 2}^{U}\right)-\sum_{j \in J}\left(v_{j 1} y_{j 1}^{L}-v_{j 2} y_{j 2}^{U}\right) \\
& \text { subject to } \\
& \underset{v_{j}}{\operatorname{maximixe}} \sum_{j \in J}\left(v_{j 1} y_{j 1}^{L}-v_{j 2} y_{j 2}^{U}\right) \\
& \text { subject to } \\
& \sum_{j \in J} S_{i j} v_{j}=0, \quad \forall i \in I \\
& L B_{j} \leq v_{j} \leq U B_{j}, \quad \forall j \in J \\
& v_{\text {biomass }} \geq v_{\text {biomass }}^{\min }, v_{\text {prod }} \geq v_{\text {prod }}^{\min } \\
& \sum_{j \in J} y_{j 1}^{L}=1, \\
& \sum_{j \in J} y_{j 2}^{U}=1 \text {, } \\
& Z \geq \varepsilon \\
& y_{j 2}^{U} \in\{0,1\}, y_{j 1}^{L} \in\{0,1\}, v_{j} \in \mathfrak{R}, \quad \forall j \in J
\end{aligned}
$$

The inner problem maximizes $v_{j_{1}}-v_{j_{2}}$ for the overproducing strain, while the outer problem maximizes the difference between a lower bound on $\min \left(v_{j_{1}}^{r e f}-v_{j_{2}}^{r e f}\right)$ [which is $\left.\min \left(v_{j_{1}}^{\text {ref,min }}-v_{j_{2}}^{\text {ref,max }}\right)\right]$ and $\max \left(v_{j_{1}}^{o s}-v_{j_{2}}^{o s}\right)$. Constraints 4 and 5 are to make sure that only one reaction is chosen for each one of $j_{1}$ and $j_{2}$. Reactions that were previously identified to belong to MUST $^{\mathrm{L}}$ and/or MUST ${ }^{\mathrm{U}}$ sets are excluded from the analysis by fixing their corresponding binary variables to zero. This optimization problem is converted to a single-level MILP similar to OptKnock and is iteratively solved to identify all reactions belonging to $\mathrm{MUST}^{\mathrm{LU}}$ sets by addition 
of integer cuts till the objective function is non-zero. This formulation can be modified to identify MUST $^{\mathrm{LL}}$, MUST ${ }^{\mathrm{UU}}$, or higher order MUST sets in a similar fashion.

\subsubsection{Identification of the FORCE sets}

Stoichiometric-based analysis of the metabolism using FBA implies that not all the flux changes encoded in the MUST sets have to be imposed in order to achieve a target yield, as many of these changes can indirectly be realized by the propagation of the effect of other interventions through the network stoichiometry. The next step of the OptForce is thus to identify the minimal set of direct interventions, referred to as FORCE sets, from the flux changes encoded by the MUST sets to achieve the desired target yield. To this end, three sets of binary variables, $y_{j}^{U}, y_{j}^{L}$ and $y_{j}^{X}$ are defined to decide whether an up-regulation, down-regulation, or reaction removal, respectively, should be chosen from the MUST set of reactions. The total number of allowed interventions is fixed at a pre-specified value $\kappa$ and FORCE sets are then identified by solving the bilevel mixed-integer optimization problem described in the following.

$$
\begin{aligned}
& \operatorname{maximize}_{y_{j}^{U}, y_{j}^{L}, y_{j}^{X}} \quad v_{\text {prod }} \\
& \text { subject to }
\end{aligned}
$$

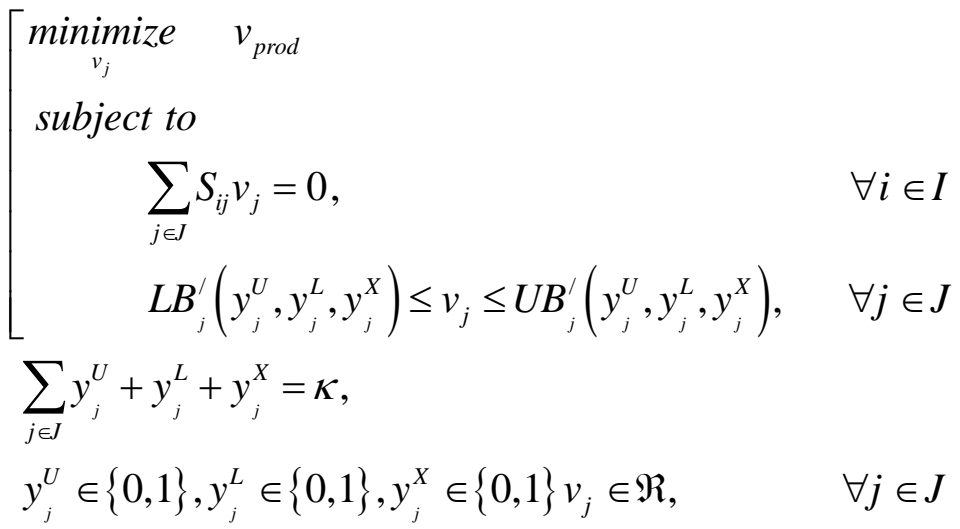

where:

$$
\begin{aligned}
& L B_{j}^{\prime}= \begin{cases}L B_{j}\left(1-y_{j}^{U}\right)+v_{j}^{o s, m i n} y_{j}^{U}, & \text { if } j \in M U S T^{U} \\
L B_{j}\left(1-y_{j}^{X}\right), & \text { if } j \in M U S T^{X} \\
L B_{j}, & \text { if } j \in J \backslash\left\{M U S T^{U} \cup M U S T^{X}\right\}\end{cases} \\
& U B_{j}^{\prime}= \begin{cases}U B_{j}\left(1-y_{j}^{L}\right)+v_{j}^{o s, m a x} y_{j}^{L}, & \text { if } j \in M U S T^{L} \\
U B_{j}\left(1-y_{j}^{X}\right), & \text { if } j \in M U S T^{X} \\
U B_{j}, & \text { if } j \in J \backslash\left\{M U S T^{L} \cup M U S T^{X}\right\}\end{cases}
\end{aligned}
$$


The outer problem, similar to OptKnock, maximizes the flux towards the target chemical by identifying flux manipulations in the network. The inner problem, however, adopts a more conservative approach assuming that the network is counteracting any flux change that is imposed by the outer problem through minimizing the production formation. If a reaction is selected to be up-regulated, the lower bound on flux is set at the minimum flux consistent with the overproducing phenotype $v_{j}^{o s, m i n}$ and it is kept at $L B_{j}$, otherwise. Likewise, if a reaction is selected for down-regulation, the upper bound on its flux is set to the maximum flux consistent with the overproducing phenotype $v_{j}^{o s, m a x}$ and to $U B_{j}$ otherwise. Finally, if a reaction is chosen for removal both lower and upper bound on its flux are set to zero. To ensure cell viability, the lower bound of biomass formation in the network is restricted to be not less than a pre-specified minimum ( $\left.v_{\text {biomass }}^{\min }\right)$, which is imposed in the inner problem. The problem is solved with the specified value of $\kappa$ to check the best achievable yield with this number of interventions. If this yield is less than the target, the value of $\kappa$ is increased incrementally (Constraint 3 ) and the optimization problem is re-solved iteratively until the desired yield is achieved. The identified interventions (when increasing the value of $\kappa$ ) often tend to be additive thereby providing a way of prioritizing the set of interventions. Alternate sets of intervention strategies are also identified by using integer cuts as before.

Similar to OptKnock, the bilevel optimization problem to identify the FORCE sets is solved by accumulating the constraints of the primal and dual of the inner problem and imposing the strong duality condition. The dual variables for constraints of the primal problem are defined as follows:

$$
\begin{array}{lll}
\sum_{j \in J} S_{i j} v_{j}=0 & \forall i \in I & \rightarrow \lambda_{i}, \text { where } \lambda_{i} \in \mathfrak{R} \\
v_{j} \geq \text { low }_{j} & \forall j \in J & \rightarrow \mu_{j}^{L B}, \text { where }_{j}^{L B} \geq 0 \\
-v_{j} \geq \text { high }_{j} & \forall j \in J & \rightarrow \mu_{j}^{U B}, \text { where }_{j}^{U B} \geq 0
\end{array}
$$

The single-level formulation for OptForce, collecting all primal and dual constraints, is described as follows: 


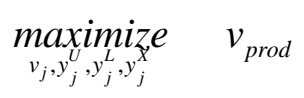

subject to

$$
\begin{array}{ll}
v_{\text {prod }}=\sum_{j \in J} \mu_{j}^{L B} L B_{j}^{\prime}-\sum_{j \in J} \mu_{j}^{U B} U B_{j}^{\prime} & \\
\sum_{j \in J} S_{i j} v_{j}=0, & \forall i \in I \\
\operatorname{low}_{j}\left(y_{j}^{U}, y_{j}^{L}, y_{j}^{X}\right) \leq v_{j} \leq \operatorname{high}_{j}\left(y_{j}^{U}, y_{j}^{L}, y_{j}^{X}\right), & \forall j \in J \\
\sum_{i \in I} \lambda_{i} S_{i j}+\mu_{j}^{L B}-\mu_{j}^{U B}=1, & \forall j \in\{\operatorname{prod}\} \\
\sum_{i \in I} \lambda_{i} S_{i j}+\mu_{j}^{L B}-\mu_{j}^{U B}=0, & \forall j \in J \backslash\{\operatorname{prod}\} \\
\sum_{j \in J} y_{j}^{U}+y_{j}^{L}+y_{j}^{X}=\kappa, & \\
\lambda_{i} \in \Re, & \forall i \in I \\
y_{j}^{U}, y_{j}^{L}, y_{j}^{X} \in\{0,1\}, \mu_{j}^{L B} \geq 0, \mu_{j}^{U B} \geq 0, v_{j} \in \mathfrak{R}, & \forall j \in J
\end{array}
$$

Note that the expressions on the right hand side of Constraint 1 are nonlinear due to the multiplication of the dual variables $\mu_{j}^{L B}$ and $\mu_{j}^{U B}$ with the binary variables $y_{j}^{L}, y_{j}^{U}$ and $y_{j}^{X}$ for the reactions in MUST sets, which can be linearized using the available standard techniques or by a customized retention of only relevant terms similarly to what was described for OptKnock.

\subsection{Simulation conditions}

The iAF1260 genome-scale model of E. coli (Feist et al., 2007) was used for all simulations. Glucose minimal conditions were simulated by restricting the glucose uptake rate to $-100 \mathrm{mmol}$ $\mathrm{gDW}^{-1} \mathrm{~h}^{-1}$ while the lower bound for the remaining exchange reactions corresponding to the metabolites present in the minimal medium was set to $-1,000 \mathrm{gDW}^{-1} \mathrm{~h}^{-1}$. Oxygen uptake was limited to zero (for fermentative conditions), and the non-growth associated ATP maintenance was fixed at $8.39 \mathrm{mmol}_{\mathrm{gDW}} \mathrm{gh}^{-1}$ (Feist et al., 2007). The upper bound on flux of the biomass reaction was set to $10 \%$ of theoretical maximum. The upper bound for all other reactions was set to 1,000 whereas the lower bound was set to zero and $-1,000$ for irreversible and reversible reactions, respectively. Metabolic flux information for 20 reactions in the central carbon metabolism (including glycolysis, TCA cycle and Pentose Phosphate pathway) (Choudhary et al., 2011) was used to define the reference phenotypic for the OptForce simulations. In addition, the overproducing phenotype was characterized to be consistent with succinate production at $90 \%$ of its theoretical maximum. Both OptKnock and OptForce were solved on an Intel Xeon X5675 3.06 
GHz processor with 48 GB RAM with a single node using the CPLEX solver accessed through GAMS.

\section{Results}

\subsection{Identified manipulations}

The suggested intervention strategies for OptKnock and OptForce procedures were contrasted for the overproduction of succinate in E. coli under anaerobic condition. Succinate is an intermediate molecule in the TCA cycle and is one of the fermentation products, which is considered as a key platform chemical for microbial production (Thakker et al., 2012). Succinate is used for the production of polymers (Xu \& Guo, 2010), industrial solvents and specialty chemicals (Budarin et al., 2007; Zeikus et al., 1999), as well as a flavoring agent in the food industry (Tsukatani \& Matsumoto, 2000). Figure 1A shows the intervention strategy suggested by OptKnock for the fermentative production of succinate in E. coli. Under anaerobic conditions, the electron transport chain is not active due to unavailability of oxygen as the terminal electron acceptor. As a result, E. coli directs metabolic flux toward fermentation products (i.e. formate, lactate, acetate, ethanol and a small amount of succinate) in order to regenerate cofactor $\mathrm{NAD}^{+}$, which is recycled back into glycolysis through glyceraldehyde-3-phosphate dehydrogenase (GAPD). OptKnock suggests four reaction removals to achieve a succinate yield of $72.3 \%$ of its theoretical maximum. These interventions remove competing $\mathrm{NAD}^{+}$regenerating pathways, such that succinate production remains the only alternative for restoring $\mathrm{NAD}^{+}$imbalance in the network. This strategy in effect couples succinate with biomass production as directed by the OptKnock bilevel optimization structure. For example, the removal of aldehyde dehydrogenase (ALCD), pyruvate formate lyase (PFL) and D-lactate dehydrogenase (LDH) prevents the metabolic flux to be diverted toward ethanol, formate and lactate production, respectively. Flux is instead rerouted towards oxaloacetate through phosphoenolpyruvate carboxylase (PPC), which is subsequently converted to succinate through the non-oxidative branch of TCA cycle active in the reverse direction. In particular, the reversal of malate dehydrogenase (MDH) and fumarase reactions (FRD) serves as the alternate $\mathrm{NAD}^{+}$regeneration system. An additional intervention suggested by OptKnock is the removal of transhydrogenase reaction (THD2pp) (Mutant A) that regenerates $\mathrm{NAD}^{+}$from the conversion of $\mathrm{NADP}^{+}$to NADPH. Removal of THD2pp prevents the network from coupling reactions that convert NADPH to $\mathrm{NADP}^{+}$with THD2pp to restore $\mathrm{NAD}^{+}$balance, thereby forcing the network to use succinate production pathway as the primary route for restoring $\mathrm{NAD}^{+}$ balance. These interventions lead to a tight coupling of growth with succinate production 
predicting a biomass formation yield of $0.012 \mathrm{gDW} / \mathrm{mmol}$ glucose and a succinate production yield of $0.79 \mathrm{gm} / \mathrm{gm}$ glucose (Mutant A, Figure 2).

Figure $1 \mathrm{~B}$ shows the intervention strategy and flux re-distribution suggested by OptForce for succinate overproduction. OptForce recapitulates some of the suggested interventions by OptKnock (such as the removal of LDH and ALCD), while also identifying a few additional interventions. In particular, OptForce suggests an at least seven-fold up-regulation (from 5 to 33.3 mmol $\mathrm{gDW}^{-1} \mathrm{~h}^{-1}$ ) of either of the reactions in the glyoxylate shunt isocitrate lyase (ICL) or malate synthase (MALS) (see Figure 3). Up-regulation of the glyoxylate shunt prevents the decarboxylation of isocitrate in the oxidative arm of TCA cycle and routes the metabolic flux toward succinate using a dual pathway one of which is directly converting isocitrate to succinate and the other redirecting the flux through the non-oxidative arm of the TCA cycle in the reverse direction, where $\mathrm{NAD}^{+}$can be regenerated. In addition, OptForce suggests a fifteen-fold (at minimum) up-regulation of PPC (from 8.4 to $133.3 \mathrm{mmol}_{\mathrm{gDW}} \mathrm{gh}^{-1}$ ) to push glycolytic flux directly to oxaloacetate, thus preventing additional flux being routed toward other fermentative products. The reversal of the non-oxidative branch of TCA cycle subsequently converts oxaloacetate and malate to succinate. PPC up-regulation along with the removal of ALCD reactions improves the minimum succinate yield from 0.216 to $0.826 \mathrm{gm} / \mathrm{gm}$ glucose (33 to 126 mmol gDW $^{-1} \mathrm{~h}^{-1}$ ). Removal of fermentative pathway reactions LDH and acetaldehyde dehydrogenase (ACALD) further increase the succinate production to $141 \mathrm{mmol} \mathrm{gDW}^{-1} \mathrm{~h}^{-1}$ (see Figure 3). These results demonstrate the impact of considering up- and/or down-regulations in addition to reaction removals in the microbial production of biochemical. It must be noted here that all the interventions suggested by OptKnock and OptForce are at the reaction level, and must be communicated to genetic level interventions using the gene-protein-reaction (GPR) relations.

Comparison with experimental studies show that the identified manipulations by both OptKnock and OptForce procedures are largely consistent with the previously proven engineering strategies (Thakker et al., 2012) for succinate overproduction in E. coli. For example, evolved E. coli strains with knock-outs for $p f l B$, $l d h$ and $a d h$ genes (encoding for PFL, LDH and ALCD reactions) have shown slight improvement in succinate production, though they suffer from $\mathrm{NAD}^{+}$cofactor imbalance (Bunch et al., 1997; Donnelly et al., 1998). As such, balance of $\mathrm{NAD}^{+}$has been identified as one of the controlling factors determining succinate production, corroborating both OptKnock and OptForce suggestions. Expression of heterologous PPC enzyme from L. lactis has improved succinate yield as well, consistent with the strategy suggested by OptForce (Lin et al., 
2005). In addition, similar to OptForce predictions, up-regulation of MDH and glyoxylate shunt, combined with the removal of fermentative pathways has shown significant improvement in succinate production (Cox et al., 2006).

\subsection{Computational performance}

Both OptKnock and OptForce become computationally expensive to solve for a large number of manipulations (e.g., four and higher). Figure 4 compares the time needed to solve OptKnock and OptForce for identifying an increasing number of interventions for the anaerobic overproduction of succinate. While the CPU times are similar for identifying a single intervention, as we move to more interventions, OptForce requires significantly less time. We performed an empirical study of both algorithms' average running time trends as a function of number of interventions. We found that whereas the running time for OptKnock varies with the cube of the number of interventions the running time for OptForce varies approximately with the square of the number of interventions. Even though OptForce is significantly more complex than OptKnock, it identifies interventions from only the MUST sets which are smaller than the complete set of reactions in the model (617 vs. 2383 for the succinate example). 


\section{Discussion}

The bilevel structure of the computational strain design algorithms such as OptKnock and OptForce helps capture the cellular objective that must be overcome to redirect flux toward the desired target. OptKnock assumes the maximization of biomass as the cellular objective, while OptForce assumes a "worst-case" scenario where the cellular metabolism actively diverts the metabolic flux away from the target product. Binary variables are used in both procedures to identify network rewiring strategies that make the target chemical an obligatory side-product of biomass formation in the former and enhance the minimal production of the target chemical in the latter. The impact of selecting the highest possible production level at maximum biomass by OptKnock can be seen in mutants A and B (Figure 1A and 2). The only difference in these two mutants is that the THD2pp removal in mutant A is replaced with the removal of glutamate dehydrogenase (GLUDy) which regenerates $\mathrm{NADP}^{+}$in mutant B. Mutant B yields a higher production of succinate $\left(125 \mathrm{mmol} \mathrm{gDW}^{-1} \mathrm{~h}^{-1}\right)$ compared to that for mutant A (123 mmol gDW ${ }^{-1}$ $\mathrm{h}^{-1}$ ). However, due to the variability in succinate production flux under the same maximum biomass production the mutants $\mathrm{A}$ and $\mathrm{B}$ are not equivalent in productivity. The removal of GLUDy does not prevent the activity of THD2pp, which can use other NADP $^{+}$producing reactions, not necessarily the succinate producing pathway, to regenerate $\mathrm{NAD}^{+}$. In contrast, succinate production is fully coupled with cellular growth for mutant A. Avoiding an optimistic production posture by OptKnock procedure was first addressed by RobustKnock (Tepper \& Shlomi, 2010), a tri-level mixed integer optimization problem that identifies reaction removals maximizing the minimum possible yield of the biomass-coupled product. An alternative approach "tilts" the inner objective function by subtracting the product flux (weighted by a small number) from biomass maximization (Feist et al., 2010) to implement the "worst-case" scenario. Note that for mutant C suggested by OptForce, succinate production happens to also be coupled with biomass formation. Both OptKnock and OptForce, however, fail to identify a number of interventions that have been found to improve succinate yield such as such as the removal of ptsG and acetate pathway reactions, and up-regulation of malic enzyme. This is because stoichiometric-based models do not capture substrate-level or other regulation information. Incorporation of substrate-level regulation was recently addressed by the k-OptForce protocol (Chowdhury et al., 2014). 
k-OptForce uses kinetic rate expressions of a subset of reactions to apportion fluxes in the rest of the metabolic network. Subsequently, similar to OptForce, a bilevel optimization problem is solved to identify up/down-regulations in the enzymatic activity of the reactions with available kinetics, along with manipulations in the stoichiometry-only part of the network to realize the targeted overproduction of desired chemical. It is to be noted here that due to the presence of the nonlinear kinetic expressions in k-OptForce formalism, a Mixed Integer Nonlinear Program (MINLP) problem must be solved (Chowdhury et al., 2014). The ultimate goal is to develop computational strain-design protocols that incorporate transcriptional, translational and posttranslational regulation in the network (McCloskey et al., 2013). Even though algorithms exist (e.g., OptORF (J. Kim \& Reed, 2010)) that can directly identify gene and transcription factor knock-outs (and expression of repressed genes) using simplified gene-to-reaction Boolean networks, we still lack the ability of using mechanistic transcriptional and translational relationships to suggest up/down-regulation of gene expressions and mRNA translation rates. Integration of synthetic biology tools (Salis, 2011; Xue et al., 2008) with systems biology metabolic engineering techniques provides an avenue for realizing this goal.

\section{Author Contributions}

Conceived and designed experiments: CDM ARZ AC. Performed the experiments: AC. Analyzed the data: AC ARZ CDM. Contributed reagents/materials/analysis tools: AC ARZ CDM. Wrote paper: AC ARZ CDM

\section{Competing Interests}

The authors have declared that no competing interests exist.

\section{Acknowledgement}

The authors gratefully acknowledge funding from the NSF (http://www.nsf.gov/) award no. EEC0813570 and the DOE (http://www.energy.gov/) grant no. DE-SC10822882. The funders had no role in the study design, data collection and analysis, decision to publish, or preparation of the manuscript. 


\section{Reference:}

Alper, H., Jin, Y. S., Moxley, J. F., \& Stephanopoulos, G. (2005). Identifying gene targets for the metabolic engineering of lycopene biosynthesis in Escherichia coli. Metab Eng, 7, 155-164.

Asadollahi, M. A., Maury, J., Patil, K. R., Schalk, M., Clark, A., \& Nielsen, J. (2009). Enhancing sesquiterpene production in Saccharomyces cerevisiae through in silico driven metabolic engineering. Metab Eng, 11, 328-334.

Bro, C., Regenberg, B., Forster, J., \& Nielsen, J. (2006). In silico aided metabolic engineering of Saccharomyces cerevisiae for improved bioethanol production. Metab Eng, 8, 102-111.

Budarin, V., Luque, R., Macquarrie, D. J., \& Clark, J. H. (2007). Towards a bio-based industry: benign catalytic esterifications of succinic acid in the presence of water. Chemistry, 13, 6914-6919.

Bunch, P. K., Mat-Jan, F., Lee, N., \& Clark, D. P. (1997). The ldhA gene encoding the fermentative lactate dehydrogenase of Escherichia coli. Microbiology, 143 ( Pt 1), 187-195.

Burgard, A. P., Pharkya, P., \& Maranas, C. D. (2003). Optknock: a bilevel programming framework for identifying gene knockout strategies for microbial strain optimization. Biotechnol Bioeng, 84, 647-657.

Choi, H. S., Lee, S. Y., Kim, T. Y., \& Woo, H. M. (2010). In silico identification of gene amplification targets for improvement of lycopene production. Appl Environ Microbiol, 76, 3097-3105.

Choudhary, M. K., Yoon, J. M., Gonzalez, R., \& Shanks, J. V. (2011). Re-examination of metabolic fluxes in Escherichia coli during anaerobic fermentation of glucose using C-13 labeling experiments and 2-dimensional nuclear magnetic resonance (NMR) spectroscopy. Biotechnology and Bioprocess Engineering, $16,419-437$.

Chowdhury, A., Zomorrodi, A. R., \& Maranas, C. D. (2014). k-OptForce: integrating kinetics with flux balance analysis for strain design. PLoS Comput Biol, 10, e1003487.

Cintolesi, A., Clomburg, J. M., \& Gonzalez, R. (2014). In silico assessment of the metabolic capabilities of an engineered functional reversal of the betaoxidation cycle for the synthesis of longer-chain $(C>/=4)$ products. Metab Eng.

Cotten, C., \& Reed, J. L. (2013). Constraint-based strain design using continuous modifications (CosMos) of flux bounds finds new strategies for metabolic engineering. Biotechnol J, 8, 595-604.

Cox, S. J., Shalel Levanon, S., Sanchez, A., Lin, H., Peercy, B., Bennett, G. N., \& San, K. Y. (2006). Development of a metabolic network design and optimization framework incorporating implementation constraints: a succinate production case study. Metab Eng, 8, 46-57.

Crown, S. B., \& Antoniewicz, M. R. (2013). Publishing 13C metabolic flux analysis studies: a review and future perspectives. Metab Eng, 20, 42-48. 
Donnelly, M. I., Millard, C. S., Clark, D. P., Chen, M. J., \& Rathke, J. W. (1998). A novel fermentation pathway in an Escherichia coli mutant producing succinic acid, acetic acid, and ethanol. Applied Biochemistry and Biotechnology, 70-2, 187198.

Famili, I., Mahadevan, R., \& Palsson, B. O. (2005). k-Cone analysis: determining all candidate values for kinetic parameters on a network scale. Biophys J, 88, 1616-1625.

Feist, A. M., Henry, C. S., Reed, J. L., Krummenacker, M., Joyce, A. R., Karp, P. D., Broadbelt, L. J., Hatzimanikatis, V., \& Palsson, B. O. (2007). A genome-scale metabolic reconstruction for Escherichia coli K-12 MG1655 that accounts for 1260 ORFs and thermodynamic information. Mol Syst Biol, 3, 121.

Feist, A. M., Zielinski, D. C., Orth, J. D., Schellenberger, J., Herrgard, M. J., \& Palsson, B. O. (2010). Model-driven evaluation of the production potential for growthcoupled products of Escherichia coli. Metab Eng, 12, 173-186.

Feng, X., Xu, Y., Chen, Y., \& Tang, Y. J. (2012). Integrating flux balance analysis into kinetic models to decipher the dynamic metabolism of Shewanella oneidensis MR-1. PLoS Comput Biol, 8, e1002376.

Fleming, R. M., Thiele, I., Provan, G., \& Nasheuer, H. P. (2010). Integrated stoichiometric, thermodynamic and kinetic modelling of steady state metabolism. J Theor Biol, 264, 683-692.

Glover, F. (1975). Improved Linear Integer Programming Formulations of Nonlinear Integer Problems. Management Science, 22, 455-460.

Ibarra, R. U., Edwards, J. S., \& Palsson, B. O. (2002). Escherichia coli K-12 undergoes adaptive evolution to achieve in silico predicted optimal growth. Nature, 420, 186-189.

Kim, J., \& Reed, J. L. (2010). OptORF: Optimal metabolic and regulatory perturbations for metabolic engineering of microbial strains. BMC Syst Biol, 4, 53.

Kim, J., Reed, J. L., \& Maravelias, C. T. (2011). Large-scale bi-level strain design approaches and mixed-integer programming solution techniques. PLoS One, 6, e24162.

Kim, T. Y., Sohn, S. B., Kim, Y. B., Kim, W. J., \& Lee, S. Y. (2012). Recent advances in reconstruction and applications of genome-scale metabolic models. Curr Opin Biotechnol, 23, 617-623.

Kumar, V. S., \& Maranas, C. D. (2009). GrowMatch: an automated method for reconciling in silico/in vivo growth predictions. PLoS Comput Biol, 5, e1000308.

Lee, S. J., Lee, D. Y., Kim, T. Y., Kim, B. H., Lee, J., \& Lee, S. Y. (2005). Metabolic engineering of Escherichia coli for enhanced production of succinic acid, based on genome comparison and in silico gene knockout simulation. Appl Environ Microbiol, 71, 7880-7887.

Lin, H., San, K. Y., \& Bennett, G. N. (2005). Effect of Sorghum vulgare phosphoenolpyruvate carboxylase and Lactococcus lactis pyruvate carboxylase coexpression on succinate production in mutant strains of Escherichia coli. Appl Microbiol Biotechnol, 67, 515-523. 
Macaulay, I. C., \& Voet, T. (2014). Single cell genomics: advances and future perspectives. PLoS Genet, 10, e1004126.

McCloskey, D., Palsson, B. O., \& Feist, A. M. (2013). Basic and applied uses of genomescale metabolic network reconstructions of Escherichia coli. Mol Syst Biol, 9, 661.

Orth, J. D., Thiele, I., \& Palsson, B. O. (2010). What is flux balance analysis? Nat Biotechnol, 28, 245-248.

Park, J. H., Lee, K. H., Kim, T. Y., \& Lee, S. Y. (2007). Metabolic engineering of Escherichia coli for the production of L-valine based on transcriptome analysis and in silico gene knockout simulation. Proc Natl Acad Sci U S A, 104, 7797-7802.

Patil, K. R., Rocha, I., Forster, J., \& Nielsen, J. (2005). Evolutionary programming as a platform for in silico metabolic engineering. BMC Bioinformatics, 6, 308.

Pharkya, P., Burgard, A. P., \& Maranas, C. D. (2004). OptStrain: a computational framework for redesign of microbial production systems. Genome Res, 14, 2367-2376.

Pharkya, P., \& Maranas, C. D. (2006). An optimization framework for identifying reaction activation/inhibition or elimination candidates for overproduction in microbial systems. Metab Eng, 8, 1-13.

Ranganathan, S., Suthers, P. F., \& Maranas, C. D. (2010). OptForce: an optimization procedure for identifying all genetic manipulations leading to targeted overproductions. PLoS Comput Biol, 6, e1000744.

Rocha, I., Maia, P., Evangelista, P., Vilaca, P., Soares, S., Pinto, J. P., Nielsen, J., Patil, K. R., Ferreira, E. C., \& Rocha, M. (2010). OptFlux: an open-source software platform for in silico metabolic engineering. BMC Syst Biol, 4, 45.

Saha, R., Chowdhury, A., \& Maranas, C. D. (2014). Recent advances in the reconstruction of metabolic models and integration of omics data. Curr Opin Biotechnol, 29C, 39-45.

Salis, H. M. (2011). The ribosome binding site calculator. Methods Enzymol, 498, 1942.

Schmidt, B. J., Ebrahim, A., Metz, T. O., Adkins, J. N., Palsson, B. O., \& Hyduke, D. R. (2013). GIM3E: condition-specific models of cellular metabolism developed from metabolomics and expression data. Bioinformatics, 29, 2900-2908.

Segre, D., Vitkup, D., \& Church, G. M. (2002). Analysis of optimality in natural and perturbed metabolic networks. Proc Natl Acad Sci U S A, 99, 15112-15117.

Suthers, P. F., Zomorrodi, A., \& Maranas, C. D. (2009). Genome-scale gene/reaction essentiality and synthetic lethality analysis. Mol Syst Biol, 5, 301.

Tepper, N., \& Shlomi, T. (2010). Predicting metabolic engineering knockout strategies for chemical production: accounting for competing pathways. Bioinformatics, 26, 536-543.

Thakker, C., Martinez, I., San, K. Y., \& Bennett, G. N. (2012). Succinate production in Escherichia coli. Biotechnol J, 7, 213-224.

Tsukatani, T., \& Matsumoto, K. (2000). Flow-injection fluorometric quantification of succinate in foodstuffs based on the use of an immobilized enzyme reactor. Analytica Chimica Acta, 416, 197-203. 
Varma, A., \& Palsson, B. O. (1994). Stoichiometric flux balance models quantitatively predict growth and metabolic by-product secretion in wild-type Escherichia coli W3110. Appl Environ Microbiol, 60, 3724-3731.

$\mathrm{Xu}$, J., \& Guo, B. H. (2010). Poly(butylene succinate) and its copolymers: research, development and industrialization. Biotechnol J, 5, 1149-1163.

Xue, X. C., Liu, F., \& Ou-Yang, Z. C. (2008). A kinetic model of transcription initiation by RNA polymerase. J Mol Biol, 378, 520-529.

Yang, L., Cluett, W. R., \& Mahadevan, R. (2011). EMILiO: a fast algorithm for genomescale strain design. Metab Eng, 13, 272-281.

Yu, D., Kim, M., Xiao, G., \& Hwang, T. H. (2013). Review of Biological Network Data and Its Applications. Genomics Inform, 11, 200-210.

Zeikus, J. G., Jain, M. K., \& Elankovan, P. (1999). Biotechnology of succinic acid production and markets for derived industrial products. Applied Microbiology and Biotechnology, 51, 545-552.

Zomorrodi, A. R., Islam, M. M., \& Maranas, C. D. (2014). d-OptCom: Dynamic Multilevel and Multi-objective Metabolic Modeling of Microbial Communities. ACS Synth Biol.

Zomorrodi, A. R., \& Maranas, C. D. (2012). OptCom: a multi-level optimization framework for the metabolic modeling and analysis of microbial communities. PLoS Comput Biol, 8, e1002363.

Zomorrodi, A. R., Suthers, P. F., Ranganathan, S., \& Maranas, C. D. (2012). Mathematical optimization applications in metabolic networks. Metab Eng, $14,672-686$. 


\section{Figure legends:}

Figure 1. Comparison of intervention strategies suggested by (A) OptKnock (Mutant A and B) and (B) OptForce (Mutant C) for overproduction of succinate in E. coli under anaerobic conditions. The values in parentheses represent the flux (in mmol $\mathrm{gDW}^{-1} \mathrm{~h}^{-1}$ ) for the corresponding reactions in the network.

Figure 2. Trade-off plot between the flux of succinate production and cellular growth rate for the reference and three mutant strains identified using OptKnock (Mutants A and B) and OptForce (Mutant C). Flux of succinate production is maximized and minimized for each mutant strain while fixing the biomass flux at a fraction of its maximum. Repeating this procedure at increasing values of biomass flux gives a plot of the feasible space of succinate production flux vs. cellular growth rate.

Figure 3. Prioritized sets of interventions suggested by OptForce and the incremental increase in succinate production yield upon addition of each set of interventions.

Figure 4. Comparison of computer wall times required for identifying increasing number of interventions for OptKnock (circles) and OptForce protocols (squares) for overproduction of succinate. 

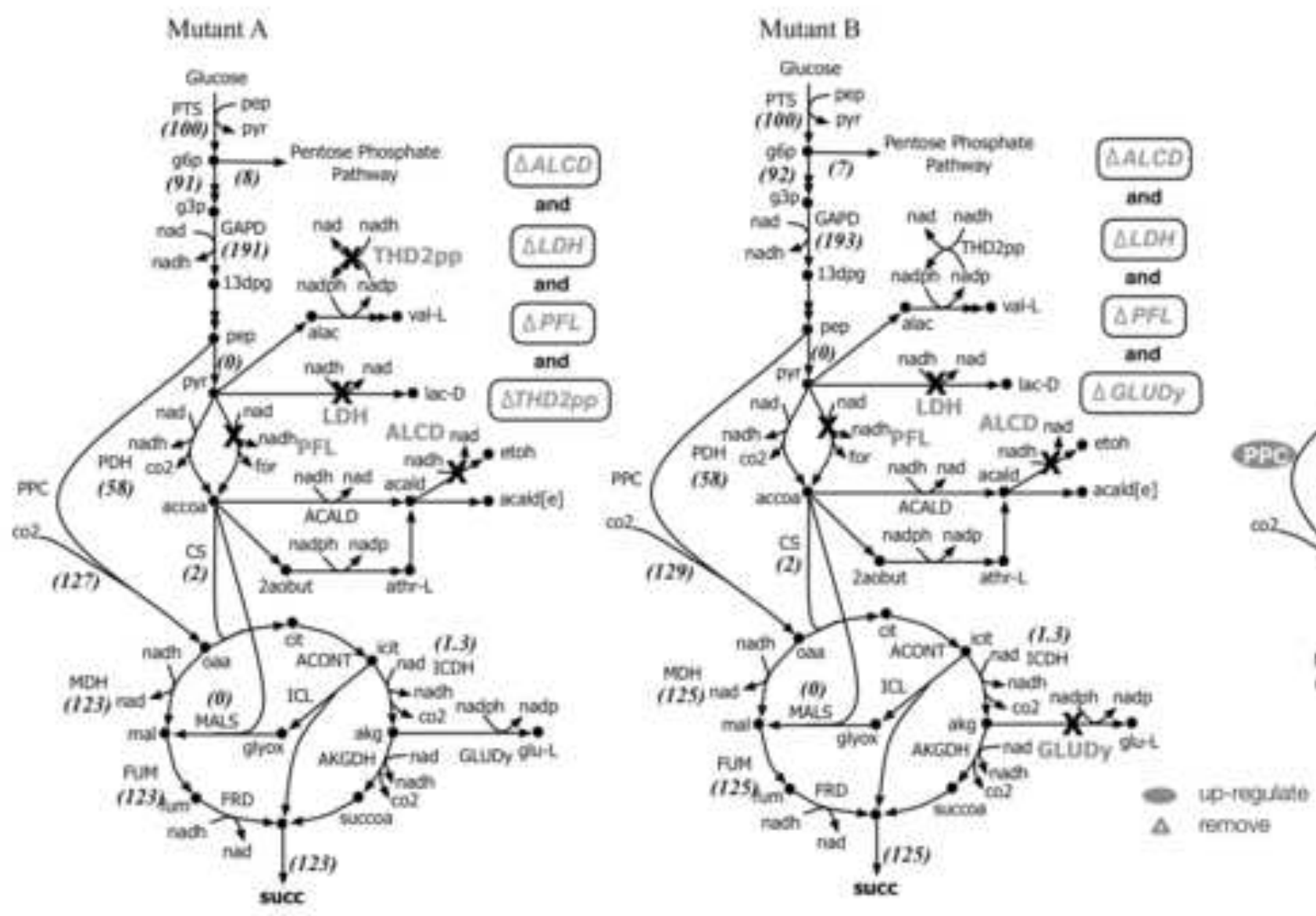

(A) OptKnock
Mutant C

Tluosese

pis t pep

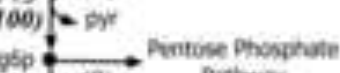

(9i)

as

nod foun was nach

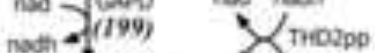

tions

- 13 esps nadón nado

$t, 1$ tones

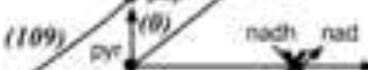

$x^{2}$

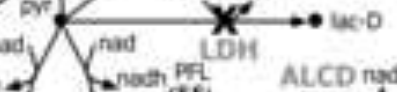

rosh 2 aroth

(19)

AcALo

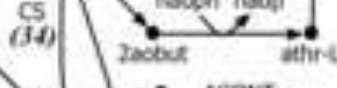

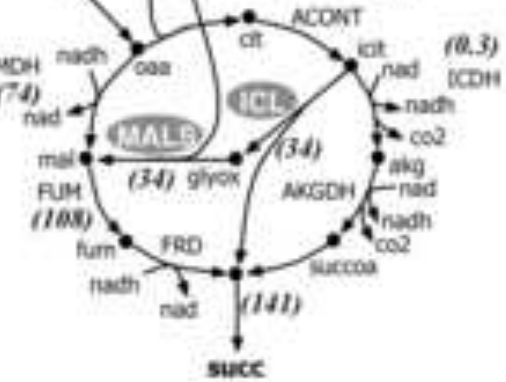

CPPC
and
$\begin{gathered}\text { Cict } \\ \text { or } \\ \text { cincs }\end{gathered}$

and

$B A L C D$

and

$\triangle \mathrm{COH}$

(B) OptForce 


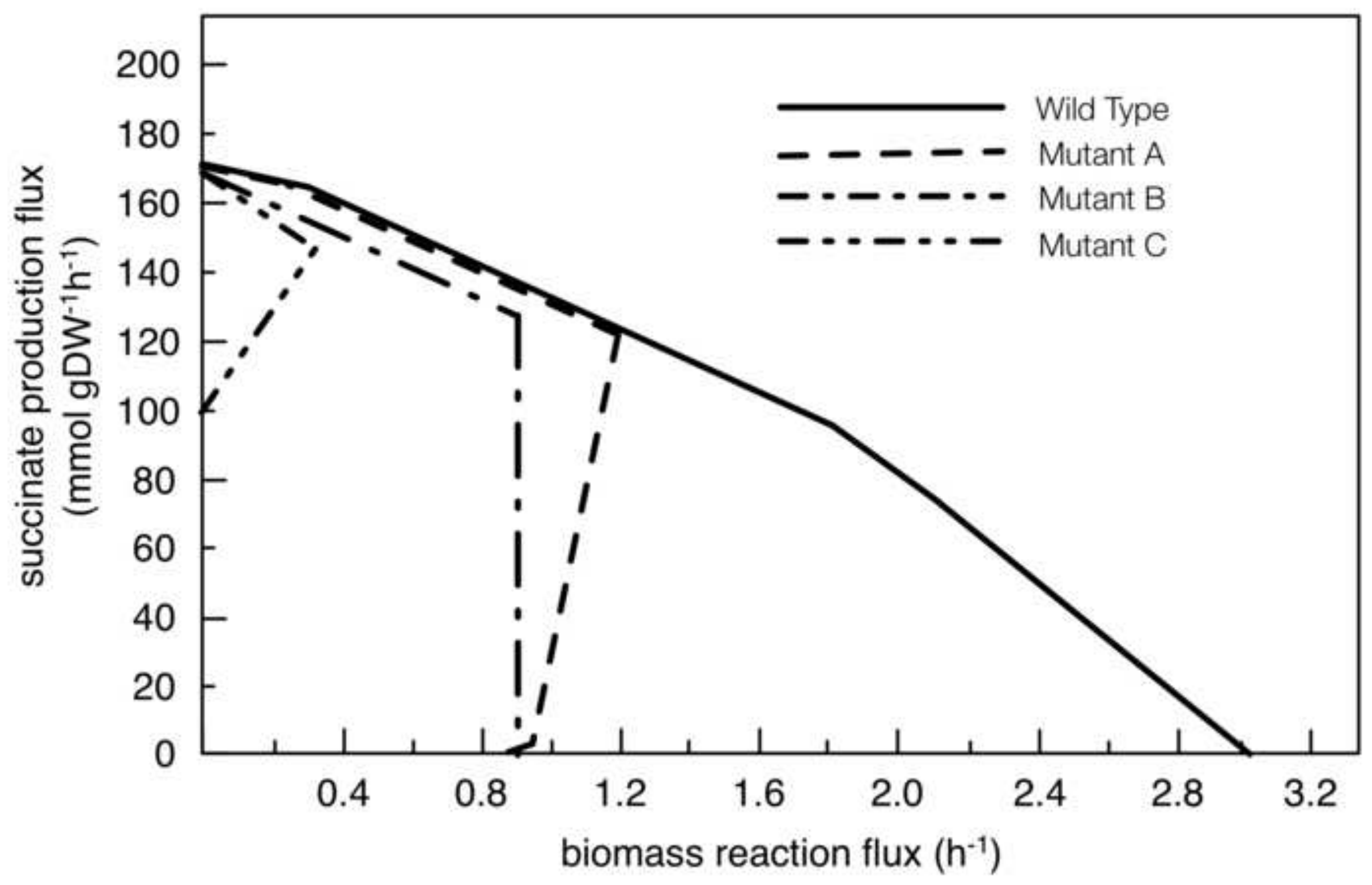




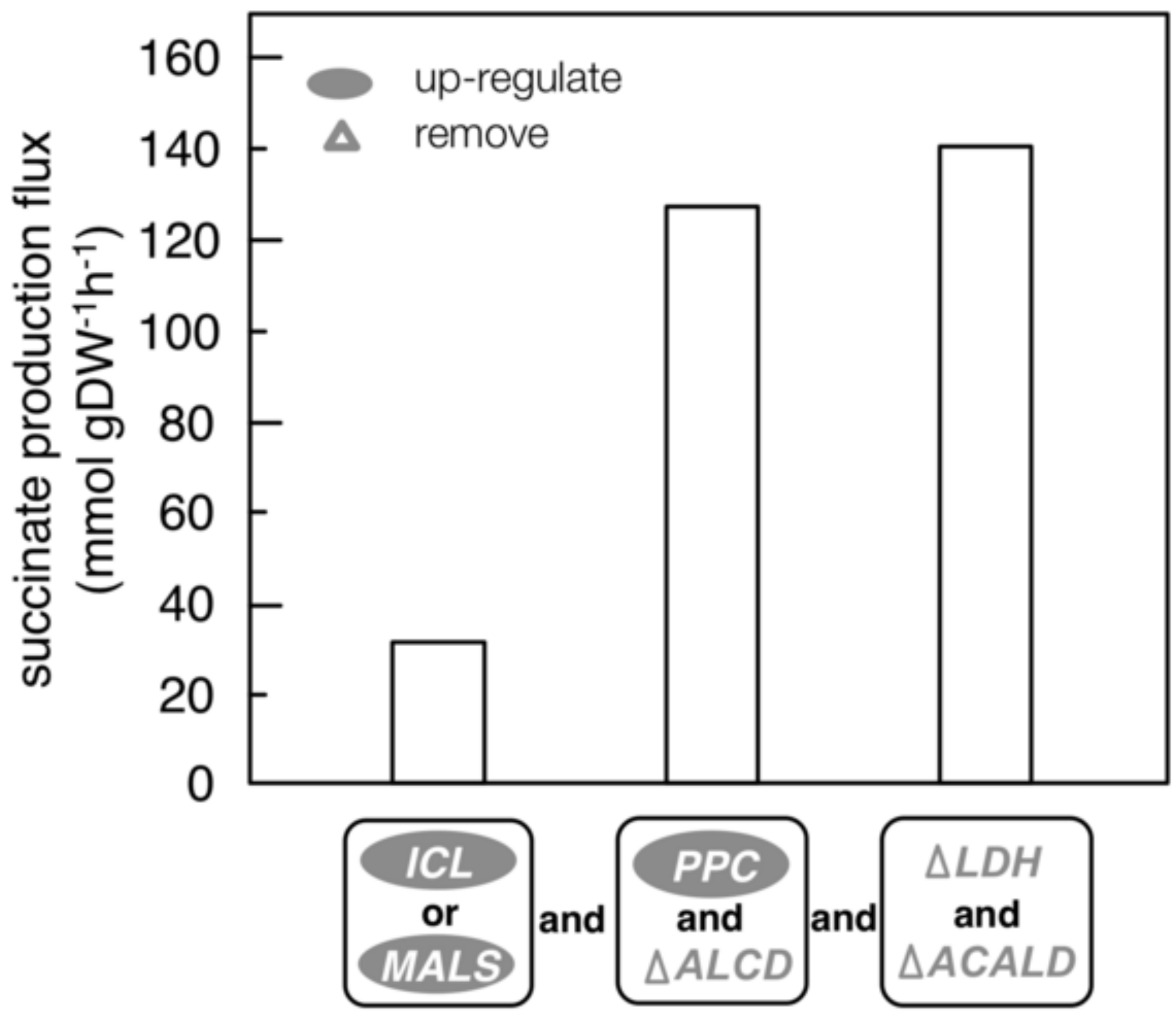

rage 31 or 32 


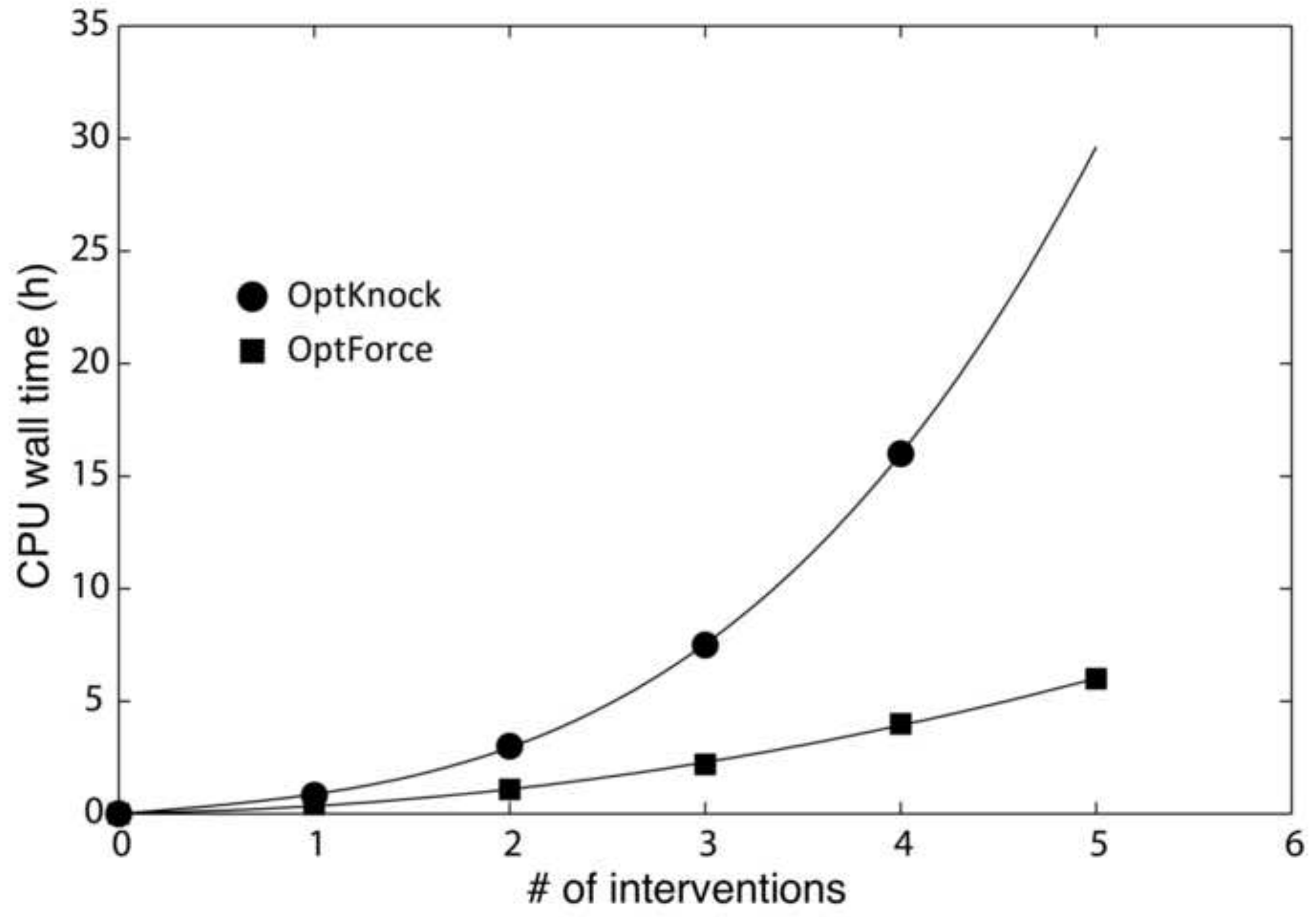

Page 32 of 32 